\title{
The Similarity of Biomarkers Level between Direct Nasal Fluid and Nasal Lavage Fluid in Allergic Rhinitis Patients
}

\author{
Jae Soon Kim, $\mathrm{MD}^{1}$, Su Jin Han, $\mathrm{MD}^{1}$, Jung Min Park, $\mathrm{RN}^{2}$, \\ Sung Wan Kim, MD, $\mathrm{PhD}^{2}$, Young-Gyu Eun, $\mathrm{MD}, \mathrm{PhD}^{2}$, and $\mathrm{Oh}$ Eun Kwon, $\mathrm{MD}, \mathrm{PhD}^{1}$ \\ ${ }^{1}$ Department of Otorhinolaryngology-Head and Neck Surgery, Kangdong Sacred Heart Hospital, Seoul; and \\ ${ }^{2}$ Department of Otorhinolaryngology-Head \& Neck Surgery, School of Medicine, Kyung Hee University, Seoul, Korea
}

Background and Objectives: Biomarkers of allergic rhinitis (AR) have been studied; however, little is known regarding their practical application in the diagnosis of AR. Previous studies collected samples using saline lavage, nasal brushing, or nasal biopsy. To utilize nasal fluid as a diagnostic tool, we need to standardize the method of sample collection. Therefore, this study aimed to evaluate the difference in concentration of biomarkers depending on the method of nasal fluid collection.

Materials and Method: Forty-five AR patients who had greater than moderate AR symptoms and who had positive results on skin prick test and serum-specific IgE tests were enrolled in this study. Nasal fluid was collected using the direct method or saline lavage method. The concentration of each biomarker was analyzed using enzyme-linked immunosorbent assay and the values compared.

Results: Nasal fluid samples were collected directly from 14 patients and were collected via saline lavage in 31 patients. No significant differences were found in the median value of each biomarker between the two methods of nasal sample collection. Conclusion: Nasal fluid collection method does not significantly affect biomarker concentration.

KEY WORDS: Allergic rhinitis · Biomarker · Diagnosis.

\section{INTRODUCTION}

Allergic rhinitis (AR) is a chronic inflammatory disease causing aggravating symptoms in patients, like nasal obstruction, watery rhinorrhea, sneezing, and itching. The pathogenesis of allergic rhinitis is associated with immunoglobulin E (IgE)-mediated immune response encompassing the release of various mediators, cytokines, and chemokines in the airway. ${ }^{1)}$ Several biomarkers for allergic rhinitis like histamine, tryptase, prostaglandin D2, mast cell-derived leukotrienes, eosinophil cationic protein (ECP), albumin, alpha2-macroglobulin, cytokines, and chemokines [interleukin (IL)-1, 3, 5, 6, 8, RANTES, and macrophage inflammatory protein-1 (MIP-1)], and nasal nitric oxide (NO) have already been identified. ${ }^{2)}$ The early and late phases of allergic response are marked by the presence of different mediators.

During the allergic reaction, inflammatory mediators promote nasal permeability by affecting the adhesion molecules of the nasal mucosa. Tryptase, a mast cell derived mediator, is increasingly present in nasal lavage fluid during AR and its levels are restored after intranasal steroid therapy. ${ }^{3 / 4)}$ Several interleukins are also associated with the pathogenesis of AR. Among them, T-helper 2 (Th2) cell derived cytokine, IL-5, is involved in the propagation and maintenance of late-phase AR. Previous studies have proposed the role of IL- 5 in AR, based on its secretion by the allergen-specific Th2 cells and also due to its elevated lev-

Received: March 16, 2021 / Revised: May 4, 2021 / Accepted: May 7, 2021

Address for correspondence: Oh Eun Kwon, MD, PhD, Department of Otorhinolaryngology-Head and Neck Surgery, Kangdong Sacred Heart Hospital, 150 Seongan-ro, Gangdong-gu, Seoul 05355, Korea

Tel: +82-2-2224-2279, Fax:+82-2-482-2279, E-mail: ononk8501@gmail.com 
els in nasal mucosal samples taken from AR patients. ${ }^{5-7)}$ The levels of MIP-1 $\beta$ in nasal mucosal samples were also found to be elevated during the late phase of AR. ${ }^{6}$ Allergic diseases might be causing changes in the airway epithelium, however, the precise mechanism behind these changes is not known. There was a study indicating an alteration in the epithelial and secretory functions of the airway epithelium during an allergic reaction. In nasal fluid, the concentration of albumin could indicate the level of epithelial permeability. ${ }^{8}$ Clara cell protein 16 (CC16), which is mainly produced by mucosal epithelial cells, has a protective role in airway inflammation, and it has been identified as a biomarker of upper and lower airway diseases. ${ }^{910)}$

Several diagnostic tools are used by clinicians for the diagnosis of allergic rhinitis, like the skin prick test (AST) or serum-specific IgE test. ${ }^{11)}$ However, these diagnostic tools have several limitations. AST has a risk of side effects and the result may be influenced by medications. ${ }^{12)}$ Serum-specific $\operatorname{IgE}$ test is convenient but it takes a long time to get the results which could be influenced by laboratory and technician factors. ${ }^{13)}$

Therefore, several biomarkers associated with AR have been explored to understand the underlying mechanism of the disease and also to assess their suitability as tools for diagnosis and monitoring of AR. ${ }^{2)}$ Based on the results of previous studies, we selected AR biomarkers, namely albumin, ECP, IL-5, tryptase, MIP-1 $\beta$, and CC16, and evaluated their efficacy for use as a tool for the diagnosis of AR. Previous studies on the identification of biomarkers of AR used samples derived from nasal mucosa biopsy, nasal brush, or nasal lavage fluid collection. ${ }^{2)}$ Our team's final purpose is to design a biosensor-based diagnostic tool using biomarkers from the nasal fluid. Depending on the patients, the clinicians can collect the nasal fluid with or without saline lavage. This study aimed to find out if there was a difference in the concentration of biomarkers when the nasal fluid was collected using two different methods: direct collection and nasal lavage fluid.

\section{MATERIALS AND METHODS}

\section{Patient selection and study design}

Patients with rhinorrhea symptoms, who tested positive in the allergy test were enrolled for this study. Patients, less than 18 years in age or those above 75 years were excluded from the study. Further, patients who were pregnant had any other sino-nasal disease or malignancy, and those suspected of cerebrospinal fluid (CSF) rhinorrhea were excluded. Thus, the AR patients included individuals who satisfied each of the following conditions: 1) having allergy symptoms such as nasal obstruction, watery rhinorrhea, itching sensation, and sneezing, and 2) positive for the allergy skin test (over $3+$ ) and multiple allergen simultaneous test (MAST) (over 2+). All participants were required to selfassess their total nasal symptoms score (TNSS) and record the results. The TNSS consisted of four major symptoms (sneezing, nasal obstruction, nasal itching, and watery nasal discharge). The degree of severity of each symptom was expressed using the following scores: $0=$ none; $1=$ =mild; $2=$ moderate; and $3=$ severe. The maximum total score was 12 points. ${ }^{14)}$

This study was approved by Kyung Hee University Medical Center Institutional Review Board before its initiation (IRB No. KHUH 2015-07-102). We received prior permission with written informed consent from all enrolled patients for their participation and the use of their samples in the study.

\section{Nasal fluid sampling and enzyme-linked immunosorbent assay (ELISA)}

Depends on the patients' condition, we collected direct nasal fluid or nasal lavage fluid from all participants. The direct nasal fluid was collected using suction from both nostrils without saline lavage and the lavage fluid was collected after instilling $1 \mathrm{~mL}, 0.9 \%$ normal saline in both nostrils. ${ }^{15)}$

All the samples were collected to a volume of over $1 \mathrm{~mL}$ using Juhn Tym Tap (Xomed Products, Jacksonville, FL) and transferred to Eppendorf tubes, and stored at $-70^{\circ} \mathrm{C}$. The measurement of the levels of albumin, CC16, tryptase, IL-5, and MIP-1 $\beta$ in nasal fluid samples was done using human-ELISA kits (Cloud-Clone Corp, Katy, TX). The nasal fluid was added to the wells of a precoated 96-well plate followed by the addition of detection reagent $\mathrm{A}$. The plate was then incubated for 1 hour at $37.8^{\circ} \mathrm{C}$ after which, the wells were washed and incubated with detection reagent B for 30 minutes at $37.8^{\circ} \mathrm{C}$. After incubation, the wells were again washed and incubated with tetramethylbenzidine (TMB) substrate solution for 15 minutes at $37.8^{\circ} \mathrm{C}$. The reaction was stopped using the stop solution, and samples were evaluated by measuring the absorbance at $450 \mathrm{~nm}$ using a microplate reader. The assays were performed in triplicates. 


\section{Statistical analysis}

Parametric data were presented as mean values with standard deviation (SD). Non-parametric grouped data were presented as median values (interquartile range, IQR) and compared using the Mann-Whitney test. Statistical significance was set at $p<0.05$. Statistical analysis of data was performed with SPSS ${ }^{\circledR}$ v 20.0 statistical software (IBM Corporation, USA).

\section{RESULTS}

In this study, $45 \mathrm{AR}$ patients (31 males and 14 females) were included. In total, 14 direct nasal fluid samples and 31 nasal lavage fluid samples were collected from all participants. The average age of the participants was $30.56 \pm$ 11.5. The mean TNSS was $8.2 \pm 2.6$, which showed moderate to severe subjective AR symptoms. The most frequently reported symptoms in TNSS were watery rhinorrhea and sneezing. The mean scores were $2.13 \pm 0.75$ and $2.2 \pm 0.75$, respectively (Table 1). There was no significant difference in patients' characteristics according to the methods of nasal fluid collection.

As shown in Table 2, we compared the levels of each parameter between direct nasal fluid samples and nasal lavage fluid samples in the AR group. The albumin, ECP, tryptase, IL-5, MIP-1 $\beta$, and CC16 were detected from both directly collected nasal fluid and nasal lavage fluid samples. The median values of albumin, ECP, tryptase, IL-5, and MIP-1 $\beta$ were similar irrespective of the sample collection method. The differences in median values of individual biomarkers were not statistically significant when the directly collected samples and nasal lavage samples were compared $(\mathrm{p}=0.08,0.52,0.27,0.47,0.51$, and 0.08 , respectively).

\section{DISCUSSION}

Biomarkers of allergic rhinitis have been studied over the years. However, the use of biomarkers as diagnostic tools is still not very popular due to the difficulties involved in procuring samples for the detection of biomarkers. Most of the studies about AR related biomarkers involve the use of nasal lavage method, nasal mucosa biopsy, or nasal brushing method. However, for using a biomarker obtained from the nasal fluid as a diagnostic tool, the method of collecting a sample must be non-invasive to increase its availability. Therefore, in this study, we attempted to determine whether there is a difference in the concentration of biomarker between samples obtained by directly collecting nasal fluid and nasal lavage fluid samples obtained using a non-invasive method. As shown in the results, we could get sufficient amounts of biomarkers from all samples regardless of the

Table 1. Demographics of participants

\begin{tabular}{lcccc}
\hline & Total $(\mathrm{n}=45)$ & Direct nasal fluid $(\mathrm{n}=14)$ & Nasal lavage fluid $(\mathrm{n}=31)$ & $\mathrm{p}$-value \\
\hline Gender (male:female) & $31: 14$ & $11: 3$ & $20: 11$ & 0.49 \\
Total serum IgE* (IU/mL) & $329.2 \pm 624.5$ & $582 \pm 876.3$ & $237.6 \pm 484.5$ & 0.27 \\
TNSS* & $8.2 \pm 2.6$ & $8.5 \pm 2.1$ & $8.3 \pm 2.7$ & 0.92 \\
Watery rhinorrhea* & $2.13 \pm 0.75$ & $2.4 \pm 0.6$ & $2.1 \pm 0.7$ & 0.6 \\
Nasal obstruction* & $1.96 \pm 1.04$ & $2.1 \pm 0.9$ & $1.75 \pm 1.15$ & 0.9 \\
Sneezing* & $2.2 \pm 0.75$ & $2.1 \pm 0.8$ & $2.3 \pm 0.6$ & 0.3 \\
Nasal itching sensation* & $2.0 \pm 0.90$ & $1.9 \pm 0.8$ & $2.1 \pm 0.8$ & 0.5 \\
\hline
\end{tabular}

*: Data presented as mean values with standard variation. AR: Allergic rhinitis, IgE: Immunoglobulin E, TNSS: total nasal symptom score

Table 2. Comparison of the concentration of biomarkers between direct collected nasal fluid and nasal lavage fluid in AR patients

\begin{tabular}{lccc}
\hline \multicolumn{1}{c}{ Parameters } & Direct nasal fluid $(\mathrm{n}=14)$ & Nasal lavage fluid $(\mathrm{n}=31)$ & $\mathrm{p}$-value \\
\hline Albumin $(\mathrm{pg} / \mathrm{mL})$ & $2125.1(630.5-6244.2)$ & $1896.0(1315.8-2817.1)$ & 0.12 \\
ECP $(\mathrm{pg} / \mathrm{mL})$ & $3909.3(-7296-73947)$ & $3649.5(2768-6195.1)$ & 0.52 \\
Tryptase $(\mathrm{pg} / \mathrm{mL})$ & $1282.2(-56.7-3474)$ & $443.5(389.8-940.9)$ & 0.27 \\
IL-5 $(\mathrm{pg} / \mathrm{mL})$ & $41.0(-2.5-131.3)$ & $23.2(25.5-72.1)$ & 0.47 \\
MIP-1 $\mathrm{beta}(\mathrm{pg} / \mathrm{mL})$ & $418.4(-68.4-14216)$ & $88.6(62.5-462.8)$ & 0.51 \\
CC16 $(\mathrm{ng} / \mathrm{mL})$ & $557.7(413.7-785.4)$ & $812.1(600.2-943.7)$ & 0.08 \\
\hline
\end{tabular}

Concentrations are expressed as median with interquartile range. AR: allergic rhinitis, ECP: eosinophil cationic protein, IL-5: Interleukin 5, MIP-1 $\beta$ : Macrophage inflammatory protein-1 $\beta, C C 16$ : Clara cell protein 
methods of sample collection. Also, there was no statistically significant difference in the concentration of biomarkers based on the collection method.

In this study, patients whose nasal fluid was collected by direct method showed severe watery rhinorrhea, although the mean score was not statistically significant. Also, the total score of TNSS was not significantly different between the two groups. However, the choice of the method of collecting nasal fluid could be influenced by the patients' dominant symptom.

Until this study, it was presumed that the saline lavage would result in a sample of lower concentration compared to the directly collected nasal fluid due to the presence of saline as a diluent. When using a nasal fluid as a biosensor, the method of collecting nasal fluid should be standardized. However, as shown in Table 2, there was no statistically significant difference in the median values of biomarkers between the directly acquired nasal fluid and the sample obtained after lavage. The result of the test could be influenced by which types of samples were used for diagnosis. Since there have been no studies on the development of an AR diagnostic kit using nasal fluid, there is no available information regarding the effects of the sample collection method on the diagnosis. However, there were some reports on the effects of sample differences on the diagnosis of other diseases and the study of microbiomes. For example, sputum is an important sample for the diagnosis of tuberculosis, and there are several methods of collecting sputum samples such as pooled sputum, spot sputum, and sputum collected in the early morning. ${ }^{16)}$ There have been several studies investigating the appropriate method of sample collection for the diagnosis of tuberculosis including a meta-analysis study that described the proper method for sputum collection. ${ }^{16)}$ Further, in the study of microbiomes, there are two commonly used methods of collecting feces which are rubbing tip and scooping into a sterile tube. ${ }^{17)}$ Research into microbiomes is still ongoing, and further research on differences in sample acquisition methods is needed. This topic requires further deliberation and hence, more studies as the method of sample collection may affect the quality of samples and eventually, the accuracy of the diagnosis.

Recently, there was a study that investigated interseasonal differences of biomarkers from nasal fluid between nonallergic subjects and seasonal AR patients. The study concluded that the nasal level of IL-8, IL-33, birch-specific IgG4, and IgE showed a significant correlation with symp- tom severity. ${ }^{18)}$ Our study included only 2 patients who were seasonal AR, so that, we think that the nasal samples from the patients might not influence the results of this study. However, in the next experiment, we need to concern about which antigens cause allergic symptoms.

There are several limitations to this study. First, the sample size was small and the number of samples in each group was different. Second, the direct and lavage nasal fluid samples were not collected from the same patients. Third, we could not get samples from control subjects because it was difficult to get enough amounts of nasal fluid samples from control subjects. However, if patients have a rhinorrhea symptom mainly, we could get the nasal sample without any additional procedure. However, we believe that our paper outweighs its limitations due to the fact that, to the best of our knowledge, this is the first trial to investigate whether a difference in the sample collection method affects the function and in turn the accuracy of an allergic rhinitis diagnostic tool. There are advantages and disadvantages to these two methods. During nasal lavage, some patients may feel discomfort while injecting normal saline into the nasal cavity, but a sufficient sample can be taken with a patient's nasal lavage. On the other hand, it may be difficult to collect a sufficient sample by directly collecting nasal fluid unless the patient has a runny nose at the time of collection.

In this study, we identified adequate concentrations of albumin, tryptase, ECP, IL-5, MIP-1 $\beta$, and CC16 from direct nasal fluid and lavage nasal fluid. Further, depending on the method of sample collection, there was no significant difference in the level of each biomarker. Therefore, both methods of collecting nasal fluid could be used in future research and diagnostic tools. Although this study has several limitations, we considered that this trial could be the first step to identify suitable biomarkers for a biosensorbased diagnostic tool of AR. In the future, we plan to compare the concentration of biomarkers between patients and healthy individuals where the latter will serve as the control group. Also, we need more studies to compare the level of biomarkers getting each method from the same patients for reinforcing this study results.

\section{REFERENCES}

1) Eifan AO, Durham SR. Pathogenesis of rhinitis. Clin Exp Allergy 2016;46(9):1139-51.

2) Diamant Z, Boot JD, Mantzouranis E, Flohr R, Sterk PJ, Gerth van Wijk R. Biomarkers in asthma and allergic rhinitis. Pulm Phar- 
macol Ther 2010;23(6):468-81.

3) de Graaf-in't Veld C, Garrelds IM, Jansen AP, Van Toorenenbergen AW, Mulder PG, Meeuwis J, et al. Effect of intranasal fluticasone proprionate on the immediate and late allergic reaction and nasal hyperreactivity in patients with a house dust mite allergy. Clin Exp Allergy 1995;25(10):966-73.

4) Boot JD, Chandoesing P, de Kam ML, Mascelli MA, Das AM, Gerth van Wijk R, et al. Applicability and reproducibility of biomarkers for the evaluation of anti-inflammatory therapy in allergic rhinitis. J Investig Allergol Clin Immunol 2008;18(6):433-42.

5) Skrindo I, Ballke C, Gran E, Johansen FE, Baekkevold ES, Jahnsen FL. IL-5 production by resident mucosal allergen-specific $\mathrm{T}$ cells in an explant model of allergic rhinitis. Clin Exp Allergy 2015; 45(8):1296-304.

6) Leaker BR, Malkov VA, Mogg R, Ruddy MK, Nicholson GC, Tan $\mathrm{AJ}$, et al. The nasal mucosal late allergic reaction to grass pollen involves type 2 inflammation (IL-5 and IL-13), the inflammasome (IL-1ß), and complement. Mucosal Immunol 2017;10(2):408-20.

7) Benson M, Strannegård IL, Wennergren G, Strannegård O. Interleukin-5 and interleukin-8 in relation to eosinophils and neutrophils in nasal fluids from school children with seasonal allergic rhinitis. Pediatr Allergy Immunol 1999;10(3):178-85.

8) Sardella A, Voisin C, Nickmilder M, Dumont X, Annesi-Maesano I, Bernard A. Nasal epithelium integrity, environmental stressors, and allergic sensitization: a biomarker study in adolescents. Biomarkers 2012;17(4):309-18.

9) Perić A, Mirković CŠ, Vojvodić D. Clara cell protein 16 release from the nasal mucosa in allergic rhinitis, chronic rhinosinusitis, and exposure to air pollutants. Arh Hig Rada Toksikol 2018;69(3):215-9.

10) Peri A, Cordella-Miele E, Miele L, Mukherjee AB. Tissue-specific expression of the gene coding for human Clara cell $10-\mathrm{kD}$ protein, a phospholipase A2-inhibitory protein. J Clin Invest 1993;92(5):2099109.

11) Scadding GK, Scadding GW. Diagnosing Allergic Rhinitis. Immunol Allergy Clin North Am 2016;36(2):249-60.

12) Corsico AG, De Amici M, Ronzoni V, Giunta V, Mennitti MC, Viscardi A, et al. Allergen-specific immunoglobulin $\mathrm{E}$ and allergic rhinitis severity. Allergy Rhinol (Providence) 2017;8(1):1-4.

13) Chen ST, Sun HL, Lu KH, Lue KH, Chou MC. Correlation of immunoglobulin E, eosinophil cationic protein, and eosinophil count with the severity of childhood perennial allergic rhinitis. J Microbiol Immunol Infect 2006;39(3):212-8.

14) Restimulia L, Pawarti DR, Ekorini HM. The Relationship between Serum Vitamin D Levels with Allergic Rhinitis Incidence and Total Nasal Symptom Score in Allergic Rhinitis Patients. Open Access Maced J Med Sci 2018;6(8):1405-9.

15) Greiff L, Pipkorn U, Alkner U, Persson CG. The 'nasal pool' device applies controlled concentrations of solutes on human nasal airway mucosa and samples its surface exudations/secretions. Clin Exp Allergy 1990;20(3):253-9.

16) Datta S, Shah L, Gilman RH, Evans CA. Comparison of sputum collection methods for tuberculosis diagnosis: a systematic review and pairwise and network meta-analysis. Lancet Glob Health 2017;5(8): e760-71.

17) Baranzini SE. Insights into microbiome research 2: Experimental design, sample collection, and shipment. Mult Scler 2018;24(11): 1419-20.

18) Gökkaya M, Damialis A, Nussbaumer T, Beck I, Bounas-Pyrros N, Bezold S, et al. Defining biomarkers to predict symptoms in subjects with and without allergy under natural pollen exposure. J Allergy Clin Immunol 2020;146(3):583-94.e6. 